\title{
The Evolution, Functions and Applications of the Breast Cancer Genes BRCA1 and BRCA2
}

\author{
CLAIRE M. PFEFFER, BENJAMIN N. HO and AMARESHWAR T.K. SINGH \\ Department of Biology, Division of Natural and Social Sciences, Carthage College, Kenosha, WI, U.S.A.
}

\begin{abstract}
BRCA1 and BRCA2 are both tumor suppressors whose mutations are the cause of most hereditary breast cancers. Both genes are highly involved in ensuring genome stability. BRCAl homologs are found in the plant and animal kingdoms while BRCA2 homologs are additionally found in the fungi kingdom. The initial origin of both genes remains unknown, however it is expected that the common ancestors originated around 1.6 billion years ago prior to the kingdoms diverging. There has been a great amount of divergence between homologs that is not observed in other tumor suppressors with only functionally important domains conserved. This divergence continues today with evidence of primate BRCA1/2 evolution. Cancer-associated mutations have been found to occur at conserved sites, indicating that conserved sites are important for function. In this study, we present a review on the phylogenesis of BRCA1 and BRCA2.
\end{abstract}

Breast cancer susceptibility genes 1 and 2 (BRCAI and BRCA2) are found in a wide variety of organisms and help stabilize the genome. BRCA2 homologs and orthologs are found in organisms across three kingdoms: animal, plant, and fungi whereas BRCAl homologs and orthologs are only found in animal and plant kingdoms. While not every organism possesses either or both of the genes, a vast majority do, indicating that the presence of $B R C A 1 / 2$ genes dates back to 1.6 billion years ago when the three kingdoms were first diverging.

Both genes are tumor suppressors and are highly involved in ensuring genome stability. BRCA1 is involved in both the

This article is freely accessible online.

Correspondence to: Dr. Amareshwar T.K. Singh, Department of Biology, Division of Natural and Social Sciences, Carthage College, Kenosha, WI, U.S.A. Tel: +1 2625516327, e-mail: asingh1@ carthage.edu

Key Words: BRCA1, BRCA2, phylogenesis, breast cancer, review. checkpoint activation and in DNA repair, while $B R C A 2$ regulates homologous recombination. $B R C A 1$ and $B R C A 2$ mutations are the cause of most hereditary breast cancer, and are responsible for up to $10 \%$ of all breast cancer. The risk for developing breast cancer is $80 \%$ with $B R C A 1$ mutations and $60 \%$ with $B R C A 2$ (1). While $B R C A 1$ and $B R C A 2$ are notoriously linked to an elevated risk of breast cancer, it has been found that they are also associated with an elevated risk for ovarian, pancreatic, endometrial, prostate, stomach, laryngeal, and fallopian tube cancers $(2,3)$. Breast cancer has been reported in other mammals including rodents, carnivores, and primates (4). This would suggest that $B R C A 1 / 2$ mutations would affect functions that are unique to mammary tissue and mammals in general, however, that is not the case. The function of both of these genes results in genome stability. $B R C A 1 / 2$ are both required for homologous recombination. $B R C A l$ is also involved in cell cycle checkpoints.

Through examining BRCA1/2 in other organisms, the functional domains can be identified that assist in determining if mutations are cancer-causing. Additionally, this allows us to learn more about the function of these genes and possible cures and treatments. Years of research and investigation of various organisms for $B R C A 1 / 2$ homologs has contributed to our knowledge of $B R C A 1 / 2$ 's role in cancer and the evolution of these genes (Table I).

\section{BRCA1 and BRCA2 Function}

The initial search for a gene responsible for hereditary breast cancer resulted in finding $B R C A 1$ that is responsible for DNA repair and checkpoints in the cell cycle (5). Further inquires into genes susceptible to breast cancer found BRCA2 which has a role in homologous recombination (6). It has been found that homozygous mutations in either of these genes is lethal and development stops embryonically. Both $B R C A 1$ and BRCA2 lead high-fidelity repair through homologous recombination (7). 
The BRCAl protein exists as a heterodimer with BARD1 and in humans, forms three different complexes. Complex A is involved in DNA repair via homologous recombination, complex B plays a role in the $\mathrm{G}_{1} / \mathrm{S}$ cell-cycle checkpoint, and complex $\mathrm{C}$ occurs in the $\mathrm{G}_{2} / \mathrm{M}$ cell-cycle checkpoint (8).

The $B R C A 2$ protein primarily functions in homologous recombination. It is responsible for recruiting RAD51 that is essential for repair. $B R C A 2$ is thought to have a special affinity for ssDNA-dsDNA junctions which are important for recombinational repair in normal replication (9). Additionally, it binds to DSS1 which assists in loading RAD51. BRCA2 also has domains that bind to both ssDNA and dsDNA (10). The main role of BRCA2 in homologous recombination is to load RAD51 onto ssDNA.

\section{Domain Structures of BRCA1 and BRCA2}

The human $B R C A 1$ protein has four major domains: RING, P300/CBP, coiled-coil, and BRCT. The RING domain interacts with BARD1 and also shows E3 ligase activity. This activity is amplified when BARD1 and BRCA1 form a heterodimer as it can ubiquitinate itself and enhance its activity (3). The P300/CBP domain has transcriptional regulatory activity. It interacts with two transcriptional cofactors: P300 (histone acetyl transferase p300) and CBP (CREB binding protein) (8). The coiled-coil domain functions in homologous recombination. The domain binds to the coiled-coil domain of PALB2 which then binds the N-terminus of BRCA2 (10). This entire complex then assists with the binding of RAD51 and continuation of homologous recombination. The BRCT domains (BRCA1 carboxy-terminal repeats) are conserved in multiple proteins that are involved in DNA repair. The BRCT domains recognize a phosphorylated serine in the pSXXF motif and bind to three different proteins: Abraxas, BRIP1, and CtIP (8). These different proteins correspond to the complexes $\mathrm{A}, \mathrm{B}$, and $\mathrm{C}$ respectively. Additionally, the BRCT domains can only bind one protein at a time, indicating that the complexes are mutually exclusive.

The human BRCA2 protein also has four domains: Nterminus, BRC, DBD, and C-terminus. As previously mentioned, the N-terminus binds to PALB2 which assists in the localization of RAD51 to the damaged DNA. The BRC repeats bind to RAD51 and assist in regulating the RAD51 filament formation (3). The DBD (DNA binding domain) contains a helix-rich region, three oligonucleotide/ oligosaccharide binding (OB) fold modules, and a tower domain (TD). Each of these regions have individual functions: OB1, OB2, and OB3 all bind ssDNA, the TD binds dsDNA, and the helix-rich region, OB1, and OB2 bind DSS1 (10). DSS1 is linked to stabilizing BRCA2, promoting homologous recombination, and has many other functions in the cell including DNA repair, development, and protein degradation (8).

\section{Phylogenesis of BRCA1 and BRCA2}

The initial origin of both BRCA1 and BRCA2 is still unknown. Both are found only in Eukaryotes; $B R C A 1 / 2$ are present in plants and animals, and $B R C A 2$ is also found in fungi. Since $B R C A 2$ is found in all three kingdoms, it is likely that the common ancestor of the gene originated prior to the kingdoms splitting around 1.6 billion years ago (11). The common ancestor of BRCAl also arose around 1.6 billion years ago when plants and animals where diverging. In order to narrow down when either gene arose, more genomes need to be annotated and examined for the functional domains in BRCA1/2.

While BRCAl homologs have been found in many eukaryotic cells, there is no recognizable one in S. cerevisiae despite the presence of some BRCA1 interacting proteins such as DSS1 (12). BRCA1 has not been identified in any other kingdom thus far, indicating that the common ancestor must be the last common ancestor of plants and animals. All plant BRCA1 homologs except green algae $C$. reinhardtii and $V$. carteri have a conserved domain, PHD, that is not present within any animal BRCA1. This suggests that there was a common BRCA1-like gene prior to plant-animal divergence that gained the PHD domain after the split. This hypothesis is confirmed by a paralog of plant BRCA1, BARD1, which also had the PHD domain. In both plants and animals, BARD1 interacts with $B R C A 1$ to form a heterodimer which is necessary for proper BRCA1 function. The plant homolog gained the PHD domain 1.15 billion years ago and the duplication to form BARD1 occurred between 495.9-301 million years ago (8).

BRCAl homologs have been found in all Viridiplanta genomes except Physcomitrella patens and in most angiosperms (8). Arabidopsis has a BRCAl homolog (AtBRCA1) that has three of the human domains: RING, BRCT, and P300/CBP (9). Throughout the entire amino acid sequence there is $20 \%$ identity between the human and Arabidopsis BRCA1, however, the identity is higher in the domains (8). As previously described, AtBRCA1 also contains the PHD domain between the P300/CBP and the BRCT domains. The function of PHD is still unknown but it is thought to be involved in the regulation of transcription (8).

A BRCA1 ortholog in C. elegans, brc-1, also functions in DNA repair and is found to be elevated during the DNA damage response (13). Overall, the function of BRCAl and its homologs have not diverged and still maintain a role in genome stability and DNA repair.

$B R C A 1$ homologs are not highly conserved, even amongst closely related species. Entire sequences have low conservation, however, some domains have higher levels of conservation. In BRCA1, a single exon, 11, encodes around $60 \%$ of the protein. This exon sequence is more viable than the entire gene for most organisms. In exon 11 within mammals, only $7.95 \%$ of the amino acid sequence is fixed 
Table I. Notable research on genes BRCA1 and BRCA2 and their evolutionary progression.

\begin{tabular}{|c|c|c|}
\hline Authors & Year & Impact \\
\hline Kojic M et al. (18). & 2002 & $\begin{array}{l}\text { BRCA2 homolog was found in Ustilago maydis, a fungus, that interacts with the Rad51 ortholog in the same } \\
\text { manner as in humans. Prior to this discovery, it was believed that only higher mammals possessed BRCA2 } \\
\text { homologs. This indicated that BRCA2 was widespread across multiple kingdoms. }\end{array}$ \\
\hline Fleming MA et al. (15). & 2003 & $\begin{array}{l}\text { Conserved regions were identified between mammalian BRCA } 1 \text { homologs. The conserved regions are all } \\
\text { located in domains that are known to interact with other proteins. Three regions were identified as being under } \\
\text { positive selection. This suggested that the BRCA1 gene is still evolving and that there is a force that is driving } \\
\text { the selection. }\end{array}$ \\
\hline Ramirez CJ et al. (4). & 2004 & $\begin{array}{l}\text { Conserved regions were identified in exon } 11 \text { of animal BRCA } 1 \text { homologs. Missense changes that are likely to } \\
\text { disrupt gene function were identified and over half of those identified occurred in highly conserved regions of } \\
\text { the gene. This indicates the importance of finding homologs and the conserved regions of genes as it can help } \\
\text { identify mutations that disrupt function and are likely disease causing. }\end{array}$ \\
\hline Dray E et al. (17). & 2006 & $\begin{array}{l}\text { The Arabidopsis ortholog of BRCA2 was shown to interact with orthologs of the proteins the human BRCA2 } \\
\text { does. The specific domains that are necessary to interact were also determined. These domains can be used to } \\
\text { evaluate possible disease causing mutations. }\end{array}$ \\
\hline Lou DI et al. (1). & 2014 & $\begin{array}{l}\text { In primates, BRCA } 1 \text { and BRCA } 2 \text { are evolving through positive selection. The hypothesis for this evolution is } \\
\text { that viral infections are driving the selection. The mutations in BRCA1 and BRCA1 make the cell less } \\
\text { susceptible to viral infection. This results in antagonistic pleiotropy. This hypothesis explains why BRCA1/2 } \\
\text { continue to evolve and pass on cancerous mutations. }\end{array}$ \\
\hline Zhou Q et al. (19). & 2014 & $\begin{array}{l}\text { The DNA binding domains of the BRCA2 ortholog in Ustilago maydis were identified and found to be similar } \\
\text { to one another yet are involved in different aspects of the repair process. Continuing to determine the function } \\
\text { of every domain of BRCA2 leads to more insights on the effects of mutations. }\end{array}$ \\
\hline
\end{tabular}

and $22.4 \%$ is conserved (4). Within exon 11 , there are 13 highly conserved regions between eutherian mammals, chicken, frog, and marsupials; three of these regions are unique to mammals (4). Since the divergence of primates from non-primates 74.1 million years ago, 132 amino acid sites in the human BRCA1 have evolved $(14,15)$.

$B R C A 1$ homologs do not share the high degree of similarity that is implied by the significant function of the gene. There is only $56 \%$ identity between the human and mouse BRCA1. Compared to other genes, this level of identity is low and displays the poor sequence conservation. MSH2 and XPA, both tumor suppressor genes, have a $92 \%$ and $86 \%$ identity, respectively, between human and mouse sequences. RAD51, which interacts with BRCA2 and is in the BRCAl complex A, has a $99 \%$ identity between human and mouse (15). Despite the low levels of conservation, it appears that function of BRCA1 has not evolved.

Similarly to $B R C A 1$, there is no $B R C A 2$ homolog in $S$. cerevisiae, however, there are homologs in other fungi. In $U$. maydis, the BRCA2 homolog is Brh2 and interacts with RAD51. Brh2 requires BRC elements in order to interact with RAD51. There is only one BRC domain in Brh2 whereas there are $8 \mathrm{BRCs}$ in the human BRCA2. Brh2 has no overall sequence alignment with any known gene, however, within the sequence are regions that align with BRCA2 conserved domains. The ability of $B R C A 2$ homologs to associate with RAD51 is conserved in U. maydis, Arabidopsis, C. elegans, and all the way up to mammals (15). In U. maydis, Brh2 functions as a master regulator of recombinational machinery. $\mathrm{Brh} 2$ is required for proficiency in repair and recombination and serves in maintaining genome stability in mitosis and meiosis (18). This function is more extensive than the human BRCA2 function suggesting that the function has narrowed and become more specified through evolution. This sequence has conservation with homologs in Arabidopsis and Oryza, with $38 \%$ identity in the $\mathrm{C}$ terminus and the nuclear localization signal (NLS). The residues that directly interact with DSS1 are the most highly conserved indicating that the interaction between them has a biologically important function (19).

Arabidopsis the is only known organism to have two BRCA2 homologs. They are $96.8 \%$ identical to each other which suggests that one arose from the other through a duplication. There is $21 \%$ identity to human BRCA2 (9). The function of these homologs has been found to be essential in meiosis. Plant homologs for BRCA2 have been found in Viridiplantae and in green algae as well. Unlike animals, plants that either lack $B R C A 2$ or are homozygous mutants are able to survive past embryonic development (8).

The chicken BRCA2 homolog has the same order and organization as the mammalian version, however, it is poorly conserved with only $37 \%$ amino acid identity. Exons 2, 4, 7, and 16-20 are highly conserved between the two homologs with an average identity of $70 \%$ (20). Any high conservation within a poorly conserved gene indicates important 
functionality. Exons 2 and 4 are in the $N$-terminal domain and exons 16-20 are in the C-terminal domain which supports $B R C A 2$ conserving function. The largest exon, 11, harbors the $\mathrm{BRC}$ repeats which are responsible for binding RAD51. In chicken $B R C A 2$, exon 11 is poorly conserved with $21 \%$ identity to human $B R C A 2$. However, three of the BRC repeats have a $70 \%$ identity between the homologs (20).

The BRC repeats are highly variable among all BRCA2 homologs. The repeats range from 1 repeat to 15 repeats in some species. The duplication of the BRC repeats happened around 230-300 million years ago (20).

Just as the BRCAl homologs are poorly conserved, the $B R C A 2$ homologs follow this same pattern. The highest conserved regions across all homologs are the BRC repeats and the C-terminal region (18). The divergence amongst the homologs could reflect the diversification and specialization in the regulation of RAD51-mediated DNA repair throughout the evolutionary tree.

\section{Human BRCA1/2 Genes}

The human BRCA1 gene is located at chromosome 17q21.3. The entire gene is 193,689 nucleotides; the coding sequence begins in the second exon and ends in the 24th exon. Exon 11 encodes more than $60 \%$ of the entire protein $(21)$. The protein is 1,863 amino acids long and $220 \mathrm{kDa}$. The BRCA2 gene is located at chromosome $12 q 13.1$. The entire gene is 91,193 nucleotides and the coding region begins in the first exon and ends in the 27th exon (22). Exon 11 encodes more than $50 \%$ of the entire protein. The BRCA2 protein is 3,418 amino acids long and $390 \mathrm{kDa}$.

\section{Limitations of Evolutionary Studies}

When looking the evolutionary relationship of $B R C A 1 / 2$, we rely on the published genomes. However, the accuracy of these genomes cannot be confirmed. Many genomes are likely to have poor assembly of the genome, or missing a percentage of the genes. Additionally, since the history of both $B R C A 1$ and $B R C A 2$ extends over one billion years, only domains or residues that are continuously selected for are truly conserved. This can lead to difficulty establishing the origin of the gene because so much divergence has occurred and can also lead to genes being included that are not true homologs, but instead just have similar domains and functions. Therefore, conclusions about the evolutionary development of either BRCA1 or BRCA2 must be taken as good estimates of past events, not as definitive statements.

\section{Relationship Between BRCA1 and BRCA2}

There is no phylogenetic relationship between $B R C A l$ and $B R C A 2$. Although $B R C A 1$ and $B R C A 2$ have similar disease phenotypes and both play a role in homologous recombination, there is no homology between each other. Neither gene shows homology to other genes in the human genome (3). Both genes are thought to have the same disease phenotype because of their joint role in homologous recombination.

\section{Continued Evolution of BRCA1 and BRCA2}

There is evidence that both $B R C A 1$ and $B R C A 2$ are still evolving, particularly in primates. It is unknown as to why both of these genes are continuing to evolve as rapidly as they are.

There are indications that there is a selection occurring at specific loci in the modern day (1). There have been 22 nonsynonymous mutations in the human BRCAl gene since the divergence from chimpanzees and bonobos six million years ago. BRCAl has far more non-synonymous mutations than other genes, indicating that it is evolving and is occurring much faster than expected. Two SNPs in the chimpanzee BRCAl were found to not be in Hardy-Weinberg equilibrium. This suggests that more than just the human $B R C A l$ gene is evolving. In simian primates, there has been a selection that favors non-synonymous mutations with BRCAl that has lasted through the speciation of the primates. The most intense selection found has occurred in the human/chimpanzee/bonobo clade (1). Most of the selection observed is positive selection. Evidence for this rapid evolution within the human BRCAl gene along with other primate homologs continues to be found across the gene (1, $4,15)$. The evolution of BRCAl in humans in occurring rapidly despite the fact that many of the changes to the gene result in a significantly elevated risk for breast cancer. Despite the rapid evolution in primate $B R C A 1$, there has been no identification of rapid evolution in marsupial BRCA1 homologs. This indicates that the selection force is acting only upon primates.

BRCA2 is also evolving under positive selection only in primates. There have been three sites in exon 11 of the human $B R C A 2$ that are under positive selection in the RAD51 interacting domain (15). The selection in BRCA2 is not concentrated in the human/chimpanzee/bonobo clade as was seen in BRCA1. This selection is not as strong as the selection observed in $B R C A 1$, but it is still occurring more rapidly than typically predicted. It remains unknown as to why these genes are evolving at this elevated rate.

The strongest hypothesis for this phenomenon is viral infections are driving this rapid evolution. Many viruses interact with DNA repair proteins in order to infect the host and remain undetected. The introduction of viral DNA can trigger the DNA damage response, yet in order to effectively infect, the virus must impede this response (23). Some viruses also use the DNA repair proteins in order to replicate themselves. These viruses could be interacting with either 
$B R C A 1$ or $B R C A 2$ and driving the changes within the genes to become less susceptible to these viruses. This selection leads to decrease vulnerability to infection, however, it also leads to consequences for the host. This scenario could lead to antagonistic pleiotropy where $B R C A 1 / 2$ are less susceptible to viral infection but increase the risk of developing breast cancer (1).

\section{Role of BRCA1 and BRCA2 in Cancer}

Many of the inherited $B R C A 1$ mutations that are cancerassociated have been found within the BRCT and RING domains which indicates that those domains are essential to the function of BRCAl (3). There have been 38 missense changes in exon 11 of BRCA 1 identified that are likely to have an effect on protein function (15). Of these 38, 3 effect fixed locations and 3 affect conservative sites as determined by the analysis of mammalian BRCAl homologs. Since the mutations affect fixed or conservative sites, they are more likely to be cancer-associated mutations as they disrupt loci that have an essential role in the protein function. The knowledge of conservative locations can be used to determine if variants are simply polymorphisms or disease associated variants. If the variant occurs at a fully or partially conserved residue, then the variant is probably diseaseassociated. Variants that have no disease link or polymorphisms typically occur in non-conserved regions.

Although mutations in both $B R C A 1$ and $B R C A 2$ increase the risk for breast cancer, they cause different cancer types. BRCA1 mutations typically result in triple-negative breast cancer (TNBC) where there is no overexpression of estrogen receptor (ER), progesterone receptor, or human epidermal growth factor receptor-2 (2). This cancer type is characterized by aggressive growth and very few targeted treatment options. The desired treatment for TNBC would only target cells with the BRCAl mutation and not healthy cells, however, since there is no overexpression, there is no target for treatment. The use of poly ADP-ribose polymerase-1 (PARP1) inhibitor and gemcitabine has shown to be a promising treatment and prompts BRCA1 mutated cells to go through apoptosis (24). Conversely, BRCA2 mutations result in low-grade $\mathrm{ER}^{+}$ luminal tumors that grow more slowly and have the treatment option of inhibiting signaling through ER (2).

While $B R C A 1 / 2$ mutations also elevate the risk of many different types of cancer, breast cancer remains the most prominent. It is still not fully understood as to why these mutations impact mammary tissue in particular, especially considering the broad role of genome stabilization that genes carry. BRCAl has been found to be a transcriptional regulator of ER; BRCA1 acts to repress the estrogen receptor which prevents further signaling that promotes cell proliferation (25). If $B R C A l$ is mutated and cannot act as a regulator, then the ER can over promote cell proliferation.
Another hypothesis is that the hormonally driven growth during the menstrual cycle produces reactive oxygen species which causes DNA damage (3). This DNA damage paired with a nonfunctional $B R C A 1$ or $B R C A 2$ leads to increased genome instability and more DNA mutations. In a study by Malicka et al. (26), it was found that physical therapy in rats lead to significantly higher progesterone levels that was found to inhibit mammary gland cancer and increase tumor cell apoptosis. This suggests that hormone treatment or physical therapy could have a suppressive effect on breast cancer development (26).

The common link between BRCAl and BRCA2 is homologous recombination. Since $B R C A 1$ and $B R C A 2$ mutations have the same disease phenotype, it can be concluded that homologous recombination is the critical system for protecting the genome. It is this pathway that is affected in both mutations, confirming that the role of $B R C A 1 / 2$ in homologous recombination is essential for tumor suppression.

\section{Conclusion}

Both the tumor suppressor genes $B R C A 1$ and $B R C A 2$ have been around for approximately 1.6 billion years in animals and plants, with BRCA2 in fungi as well. Much of our knowledge about both of these genes have come from studying homologs in various organisms. Additionally, the realization that both $B R C A 1$ and $B R C A 2$ are still rapidly evolving in primates explains the perseverance of disease causing mutations in the genes.

\section{Conflicts of Interest}

The Authors declare no competing financial interests.

\section{Acknowledgements}

The Authors gratefully acknowledge the support from the Department of Biology, Division of Natural and Social Sciences, Carthage College, Kenosha, WI 53140 for supporting Claire Pfeffer and Benjamin Ho as Fellows to conducting research under the mentorship of Dr. Amareshwar T.K. Singh.

\section{References}

1 Lou DI, McBee RM, Le UQ, Stone AC, Wilkerson GK, Demogines AM and Sawyer SL: Rapid evolution of BRCA1 and BRCA2 in humans and other primates. BMC Evol Biol 14: 155, 2014.

2 Talens F, Jalving M, Gietema JA and Van Vugt MA: Therapeutic targeting and patient selection for cancers with homologous recombination defects. Expert Opin Drug Discov 12(6): 565$581,2017$.

3 Roy R, Chun J and Powell SN: BRCA1 and BRCA2: different roles in a common pathway of genome protection. Nat Rev Cancer 12(1): 68-78, 2016. 
4 Ramirez CJ, Fleming MA, Potter JD, Ostrander GK and Ostrander EA: Marsupial BRCA1: conserved regions in mammals and the potential effect of missense changes. Oncogene 23: 1780-1788, 2004.

5 Hall JM, Lee MK, Newman B, Morrow JE, Anderson LA, Huey $B$ and King MC: Linkage of early-onset familial breast cancer to chromosome 17q21. Science 250: 1684-1689, 1990.

6 Wooster R, Bignell G, Lancaster J, Swift S, Seal S, Mangion J, Collins N, Gregory S, Gumbs C and Micklem G: Identification of the breast cancer susceptibility gene BRCA2. Nature 378 : 789-792, 1995.

7 Muggia F and Safra T: 'BRCAness' and its implications for platinum action in gynecologic cancer. Anticancer Res 34(2): 551-556, 2014.

8 Trapp O, Seeliger K and Puchta H: Homologs of breast cancer genes in plants. Front Plant Sci 2(19): 1-17, 2011.

9 Seeliger K, Dukowic-Schulze S, Wurz-Wildersinn R, Pacher M and Puchta H: BRCA2 is a mediator of RAD51- and DMC1facilitated homologous recombination in Arabidopsis thaliana. New Phytologist 193: 364-375, 2012.

10 Prakash R, Zhang Y, Feng W and Jasin M: Homologous recombination and human health: the roles of BRCA1, BRCA2 and associated proteins. Cold Spring Harb Perspect Biol 7(4): a016600, 2015.

11 Wang DY, Kumar S and Hedges SB: Divergence time estimates for the early history of animal phyla and the origin of plants, animals and fungi. Proc Biol Sci 266: 163-171, 1999.

12 Golubeva V and Monteiro AN: What can yeast tell us about breast cancer? Cell cycle 12(2): 157-158, 2017.

13 Labocha MK, Yuan W, Aleman-Meza B and Zhong W: A strategy to apply quantitative epistasis analysis on developmental traits. BMC Genetics 18: 42, 2017.

14 Pozzi L, Hodgson JA, Burrell AS, Sterner KN, Raaum RL and Disotell TR: Primate phylogenetic relationships and divergence dates inferred from complete mitochondrial genomes. Mol Phylogenet Evol 75: 165-183, 2014.

15 Fleming MA, Potter JD, Ramirez CJ, Ostrander GK and Ostrander EA: Understanding missense mutations in the BRCA1 gene: an evolutionary approach. Proc Natl Acad Sci 100(3): 1151-1156, 2003.

16 Jasin M: Homologous repair of DNA damage and tumorigenesis: the BRCA connection. Oncogene 21: 8981-8993, 2002.
17 Dray E, Siaud N, Dubois E and Doutriaux MP: Interaction between Arabidopsis Brca2 and its partners Rad51, Dmc1, and Dss1. Plant Physiol 140(3): 1059-1069, 2006.

18 Kojic M, Kostrub CF, Buchman AR and Holloman WK: BRCA2 homolog required for proficiency in DNA repair, recombination, and genome stability in Ustilago maydis. Mol Cell 10(3): 683691, 2002.

19 Zhou Q and Holloman WK: Dual DNA-binding domains shape the interaction of Brh2 with DNA. DNA repair 22: 104-111, 2014.

20 Warren M, Smith A, Partridge N, Masabanda J, Griffin D and Ashworth A: Structural analysis of the chicken BRCA2 gene facilitates identification of functional domains and disease causing mutations. Hum Mol Genet 11(7): 841-851, 2002.

21 Orban TI and Olah E: Emerging roles of BRCA1 alternative splicing. Mol Pathol 56(4): 191-197, 2003.

22 Mehta PA and Tolar J: Fanconi Anemia. In: GeneReviews [Internet]. Pagon RA, Adam MP, Ardinger HH, Dinulos MB and Wallace SE (eds.). Seattle, University of Washington, Seattle, 2004.

23 Demogines A, East AM, Lee JH, Grossman SR, Sabeti PC, Paull TT and Sawyer SL: Ancient and recent adaptive evolution of primate non-homologous end joining genes. PLoS Genet 6(10): e1001169, 2010.

24 Sasaki A, Tsundoa Y, Tsuji M, Udaka Y, Oyamada H, Tsuchiya $\mathrm{H}$ and Oguchi K: Decreased miR-206 expression in BRCA1 wild-type triple-negative breast cancer cells after concomitant treatment with gemcitabine and a poly(ADP-ribose) polymerase1 inhibitor. Anticancer Res 34(9): 4893-4897, 2014.

25 Wang L and Di LJ: BRCA1 and estrogen/ estrogen receptor in breast cancer: where they interact? Int J Biol Sci 10(5): 566-575, 2014.

26 Malicka I, Siewierska K, Kobierzycki C, Grzegrzolka J, Piotrowska A, Paslawska U, Cegielski M, Dziegiel P, PodhorskaOkolow P and Wozniewski M: Impact of physical training on sex hormones and their receptors during $N$-methyl- $N$ nitrosourea-induced carcinogenesis in rats. Anticancer Res 37(7): 3581-3589, 2017.
Received July 5, 2017

Revised July 26, 2017

Accepted August 2, 2017 